\title{
Interaction network of coexpressed mRNA, miRNA, and IncRNA activated by TGF- $\beta 1$ regulates EMT in human pulmonary epithelial cell
}

\author{
HUIZHU LIU ${ }^{1 *}$, XUEYING ZHAO ${ }^{2 *}$, JING XIANG $^{3 *}$, JIE ZHANG $^{1}, \mathrm{CHAO} \mathrm{MENG}^{4}$, \\ JINJIN ZHANG ${ }^{1}$, MINGE $\mathrm{LI}^{2}$, XIAODONG SONG ${ }^{1}$ and CHANGJUN LV ${ }^{1,4}$ \\ ${ }^{1}$ Department of Cellular and Genetic Medicine, School of Pharmaceutical Sciences, Binzhou Medical \\ University, Yantai $264003 ;{ }^{2}$ Department of Clinical Nursing, Affiliated Hospital to Binzhou Medical University, \\ Binzhou 256602; ${ }^{3}$ Department of Statistics, Binzhou Medical University, Yantai 264003; ${ }^{4}$ Department of \\ Respiratory Medicine, Affiliated Hospital to Binzhou Medical University, Binzhou 256602, P.R. China
}

Received April 6, 2017; Accepted September 15, 2017

DOI: $10.3892 / \mathrm{mmr} .2017 .7653$

\begin{abstract}
Noncoding RNAs (ncRNAs), such as microRNAs (miRNAs) and long noncoding RNAs (lncRNAs), play increasingly important roles in pathological processes involved in disease development. However, whether mRNAs interact with miRNAs and lncRNAs to form an interacting regulatory network in diseases remains unknown. In this study, the interaction of coexpressed mRNAs, miRNAs and lncRNAs during tumor growth factor- $\beta 1$-activated (TGF- $\beta 1$ ) epithelial-mesenchymal transition (EMT) was systematically analyzed in human alveolar epithelial cells. For EMT regulation, 24 mRNAs, 11 miRNAs and 33 lncRNAs were coexpressed, and interacted with one another. The interaction among coexpressed mRNAs, miRNAs and lncRNAs were further analyzed, and the results showed the lack of competing endogenous RNAs (ceRNAs) among them. The mutual regulation may be correlated with other modes, such as histone modification and transcription factor recruitment. However, the possibility of ceRNA existence cannot be ignored because of the generally low abundance of lncRNAs and frequent promiscuity of protein-RNA interactions. Thus, conclusions need further experimental identification and validation. In this context, disrupting many altered disease pathways remains one of the challenges in obtaining effective pathway-based therapy. The reason being that one specific mRNA, miRNA or lncRNA may target multiple genes that are
\end{abstract}

Correspondence to: Professor Xiaodong Song or Professor Changjun Lv, Department of Cellular and Genetic Medicine, School of Pharmaceutical Sciences, Binzhou Medical University, 346 Guanhai Road, Yantai 264003, P.R. China

E-mail: songxd71@163.com

E-mail: lucky_lcj@sina.com

*Contributed equally

Key words: mRNA, microRNAs, long noncoding RNAs, competing endogenous RNAs, epithelial-mesenchymal transition potentially implicated in a disease. Nevertheless, the results of the present study provide basic mechanistic information, possible biomarkers and novel treatment strategies for diseases, particularly pulmonary tumor and fibrosis.

\section{Introduction}

Epithelial-mesenchymal transition (EMT) endows cells with increased motility and invasiveness during the development of diseases, especially tumor and fibrosis $(1,2)$. Tumor growth factor (TGF) $\beta$ can boost tumor or fibrosis progression by enhancing proliferation, migration, and invasion partly because it can induce EMT $(3,4)$. However, the underlying molecular mechanisms that mediate EMT remain unclear. Thus, enhancing our understanding of these molecular mechanisms lead to the development of effective EMT-targeted therapy and improvement of the diagnosis, treatment, and prognosis of tumor or fibrosis $(5,6)$.

Emerging studies showed that noncoding RNAs (ncRNAs), such as microRNAs (miRNAs) and long noncoding RNAs (lncRNAs), participate in many critical biological processes, such as cell proliferation, apoptosis, and differentiation (7-9). Furthermore, the regulatory network of ncRNAs participates in the development of various illnesses; in particular, miRNAs inhibit the crucial process of target RNA transcription (10-13), and lncRNAs exhibit facilitative or suppressive effects on the gene-regulatory network (14-16). However, the relationships among messenger RNAs (mRNAs), miRNAs, and lncRNAs remain unclear. A competing endogenous RNA (ceRNA) mechanism that partly explains their mutual regulation of mRNAs, miRNAs, and lncRNAs is hypothesized to occur in a disordered manner. Furthermore, lncRNAs, such as GAS5 and glucocorticoid receptor (18), GADD7 and TDP-43 (19), and PANDA and NF-YA (20), that inhibit proteins through competitive binding miRNA were reported (17). Nevertheless, owing to the generally low abundance of lncRNAs and frequent promiscuity of protein-RNA interactions, the extent to which lncRNAs function through this mechanism is highly debated (21). 
Thus, characterizing the interactions among mRNA, miRNA, and lncRNA can enhance our understanding of disease pathogenesis. Our previous studies reported different lncRNA profiles in bleomycin-induced rats $(22,23)$. However, the rats' genes showed a high degree of difference from its human homologs at IncRNA, miRNA, and mRNA levels. Thus, their interactions cannot be analyzed completely. In addition, TGF- $\beta 1$ is the prototypic growth factor for the induction of EMT-like changes in tumor or fibrosis $(1,2)$. Thus, determining what changes can occur during the interactions among mRNAs, miRNAs, and lncRNAs upon TGF- $\beta 1$ stimulation during EMT is important. The A549 cell line displays properties similar to those of human type II pulmonary epithelial cells (24) and is thus often used as the normal model for studying pulmonary fibrosis or other pulmonary diseases (25-27). In the present study, the interactions among coexpressed mRNAs, miRNAs, and IncRNAs were assessed in TGF- $\beta 1$-activated EMTs of A549 cells. The results demonstrated that networks mediated by EMT-associated mRNAs, miRNAs, and lncRNAs can be used to accelerate the discovery of molecular mechanisms and EMT-targeted therapeutics for pulmonary tumor and fibrosis.

\section{Materials and methods}

Cell model. A549 and MRC-5 cell lines were purchased from Cell Bank of Chinese Academy of Sciences (Shanghai, China). Cells were maintained in Dulbecco's modified Eagle's medium and advanced minimum essential medium, respectively, supplemented with $10 \%$ newborn calf serum, $100 \mathrm{U} / \mathrm{ml}$ penicillin, and $100 \mu \mathrm{g} / \mathrm{ml}$ streptomycin at $37^{\circ} \mathrm{C}$ under a humidified atmosphere of $5 \% \mathrm{CO}_{2}$ and $95 \%$ air. A549 or MRC-5 cells were collected after treatment with $10 \mathrm{ng} / \mathrm{ml}$ TGF- $\beta 1$ for 12 , 24 , and $48 \mathrm{~h}$ as our previously described (28-30).

Differentially expressed mRNAs, miRNAs and IncRNAs. Total RNA was harvested and quantified, and its integrity was verified by denaturing gel electrophoresis. Samples with a 28S:18S ratios of approximately 2:1 were accepted for further analysis, which was performed on an Affymetrix Human Transcriptome Array 2.0 microarray for mRNAs and lncRNAs and Affymetrix Gene Chip miRNA 4.0 microarray for miRNAs. The detection of mRNAs, miRNAs, and lncRNAs was performed according to the manufacturer's instructions. Finally, differentially expressed mRNAs, miRNAs, and IncRNAs were identified through fold-change filtering after the quantile normalization of raw data.

Bioinformatics analysis of differentially expressed $m R N A$. Gene ontology (GO) analysis was applied to predict the main functions of the target genes according to the GO project. Fisher's exact test and $\chi^{2}$ were used to classify the GO category. Moreover, false discovery rate (FDR) was calculated to correct the P-value. The smaller the FDR, the smaller the error in judging the P-value. The standard difference screening was $\mathrm{P}<0.01$.

Pathway analysis was performed to determine the significant pathway of the differential genes according to the Kyoto Encyclopedia of Genes and Genomes (KEGG), Biocarta, and Reatome. Fisher's exact test and $\chi^{2}$ results were used to select the significant pathway, and the significance threshold was defined by P-value and FDR. The standard difference screening was $\mathrm{P}<0.05$.

MiRNA-target gene network was established on the basis of GO and KEGG predicted data for the illustration of the relationship between miRNAs and their target genes.

Quantitative real-time PCR ( $q R T$-PCR). Total RNA was isolated with TRIzol reagent (Invitrogen, Carlsbad, CA, USA). RNA quantity and quality were measured on a NanoDrop 2000 spectrophotometer (Thermo Fisher Scientific, Waltham, MA, USA). RNA integrity was assessed by standard denaturing agarose gel electrophoresis. Complementary DNA synthesis was performed with a Moloney Murine Leukemia Virus reverse transcriptase kit (Invitrogen) following the manufacturer's instructions. qRT-PCR was performed with a SYBR-Green-based PCR master mix kit (Takara, Shiga, Japan) on a Rotor Gene 3000 real-time PCR system from Corbett Research (Sydney, Australia). Primers of lncRNAs as the following: linc00941 sense: 5'-GCGGTA GCCTTCTCTGAACTG-3', antisense: 5'-GTTGCATAACCT GACCTGCC-3'; AF086191 sense: 5'-GCAGAGTGGAGCCTT CTCAT-3', antisense: 5'-TATGCAAACTCCCATGTGGC-3'. Quantification of miR-500a-5p, miR-628-3p, miR-128-3p, miR-223-3p and miR-30a was performed with a stem-loop real-time PCR miRNA kit bought from Guangzhou RiboBio Co., Ltd. (Guangzhou, China).

Western blot analysis. Protein concentration was quantified using a bicinchoninic acid protein assay kit and boiled with the sample buffer in a water bath for 5 min. Protein samples were separated with $15 \%$ SDS-PAGE gels for $2 \mathrm{~h}$ and transferred onto a polyvinylidene difluoride membrane, which was subsequently blocked in 5\% non-fat milk for $2 \mathrm{~h}$. Blots were probed using the primary antibodies. The anti-vimentin, a-SMA, E-cadherin and Snail antibody was from ProteinTech Group. After three times washing with tris buffered saline tween, the horseradish peroxidase-conjugated secondary antibodies were added. Antigen-antibody complexes were visualized by enhanced chemiluminescence.

Transmission electron microscopy (TEM) observation. Cells were fixed by treatment with fresh $2.5 \%$ glutaraldehyde at $4^{\circ} \mathrm{C}$ for at least $4 \mathrm{~h}$, post-fixed in $1 \%$ osmium tetroxide for $1.5 \mathrm{~h}$, dehydrated in gradient ethanol, infiltrated with Epon812, embedded and cultured at $37^{\circ} \mathrm{C}$ for $12 \mathrm{~h}, 45^{\circ} \mathrm{C}$ for $12 \mathrm{~h}$ and $60^{\circ} \mathrm{C}$ for $24 \mathrm{~h}$. Ultrathin sections prepared with an ultracut $\mathrm{E}$ ultramicrotome were stained with uranyl acetate and lead citrate and observed using a JEM-1400 TEM (JEOL Ltd., Tokyo, Japan).

Statistical analysis. All data are expressed as the means \pm SD. Statistical analysis was performed using SPSS13.0 software by one-way ANOVA. $\mathrm{P}<0.05$ was considered to indicate a statistically significant difference.

\section{Results}

Identification of the cell model. EMT is the phenomenon in which epithelial cells obtain mesenchymal characteristics, including change in shape, expression of mesenchymal markers, such as collagen and a smooth muscle actin (a-SMA); loss of epithelial cell marker E-cadherin; and increase of 

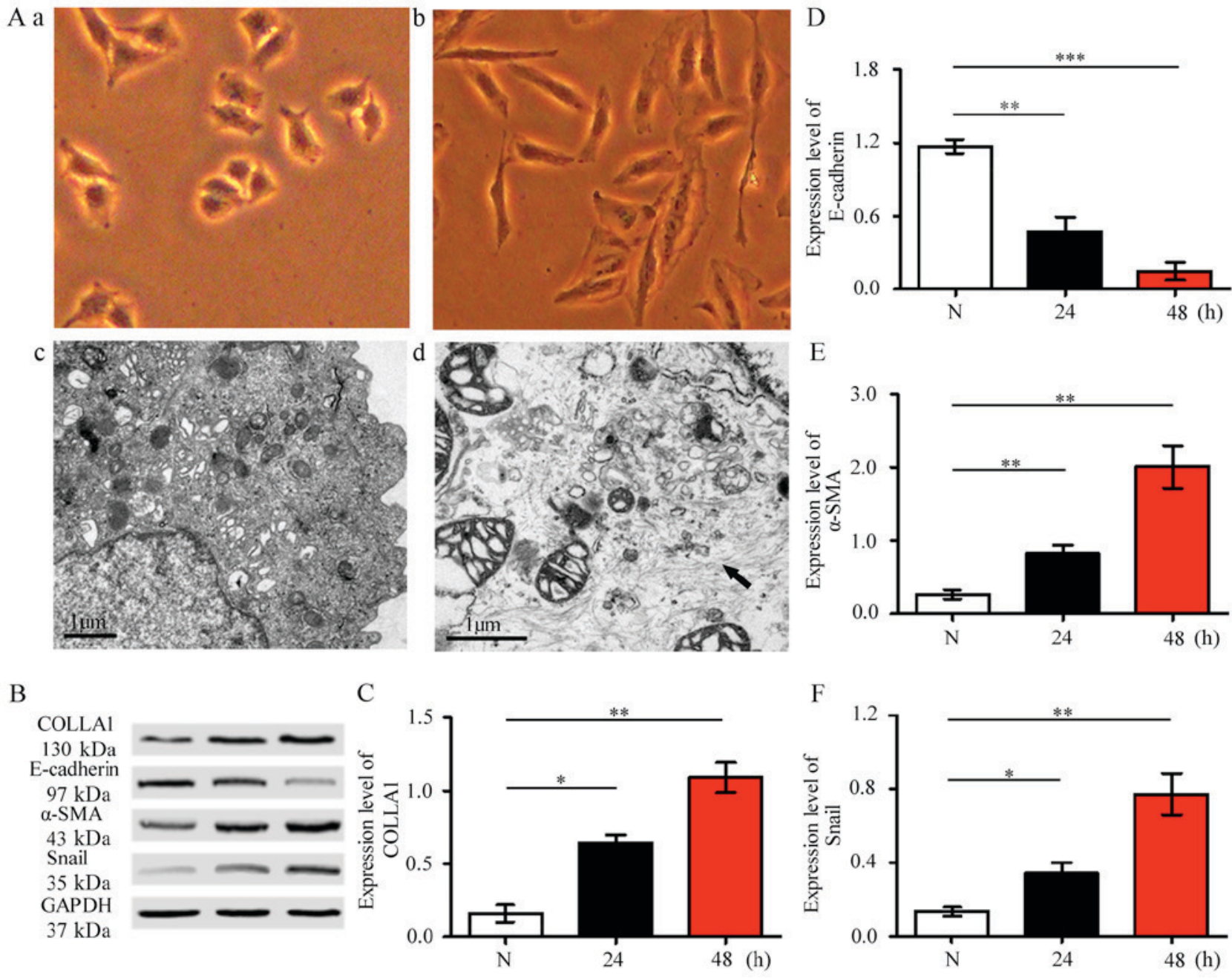

Figure 1. Identification of cell model. (A) Optical microscopy observed A549 cell shape changed from irregular to spindle (a and b), x400 magnification. TEM image showed more muscle fibers in the cytoplasm of TGF- $\beta 1$-activated cells compared with those in normal controls (c and d). (B-F) Expression levels of collagen, a-SMA, E-cadherin, and Snail were determined using western blot analysis in A549 cell. The expression levels of collagen I, a-SMA, and Snail significantly increased, whereas that of E-cadherin significantly decreased. Data are expressed as means $\pm \mathrm{SD},{ }^{*} \mathrm{P}<0.05,{ }^{* *} \mathrm{P}<0.01,{ }^{* * * *} \mathrm{P}<0.001$ vs. control. TEM, transmission electron microscopy; TGF- $\beta 1$, tumor growth factor- $\beta 1$; SMA, smooth muscle actin.

transcription repressor Snail. Under an optical microscope, the shapes of the cells changed from irregular to spindle. In the TEM images, the number of muscle fibers in the cytoplasm of TGF- $\beta 1$-activated cells are larger than those of normal control cells (Fig. 1A). Meanwhile, the expression levels of collagen I, a-SMA, E-cadherin, and Snail were significantly increased, whereas that of E-cadherin expression significantly decreased (Fig. 1B-F). These findings indicated the successful establishment of the cell model.

Differentially coexpressed mRNAs, miRNAs, and IncRNAs. The expressed profiles were determined by using Affymetrix Human Transcriptome Array 2.0 microarray for mRNAs and lncRNAs and Affymetrix Gene Chip miRNA4.0 microarray for miRNAs in A549 and TGF- $\beta 1$-activated A549 at 12, 24, and 48 h, respectively. Hierarchical clustering analysis results showed that the mRNAs, miRNAs, and lncRNAs of the cell models are differentially expressed (Fig. 2A-C). Basing on our previous studies and other published works, we analyzed the data of TGF- $\beta 1$-activated A549 only at 24 and $48 \mathrm{~h}$. A total of 242 mRNAs were differentially coexpressed in TGF- $\beta 1$-activated 24 and $48 \mathrm{~h}$ cell samples compared with the normal control group. Of these mRNAs, 139 were upregulated, 103 were downregulated, and 143 miRNAs were differentially coexpressed in TGF- $\beta 1$-activated 24 and $48 \mathrm{~h}$ cell samples compared with the normal control group. Among these miRNAs, 83 were upregulated, 60 were downregulated, and 127 lncRNAs were differentially coexpressed in TGF- $\beta 1$-activated 24 and $48 \mathrm{~h}$ cell samples compared with the normal control group. Among which, 71 were upregulated and 56 were downregulated.

To validate the microarray data, we randomly selected two miRNAs (miR-500a-5p and miR-628-3p) and two lncRNAs (linc00941 and AF086191) and determined their expression levels through qRT-PCR. These RNAs were selected because they have relatively higher differences than other RNAs. As shown in Fig. 2, the two miRNAs and two lncRNAs were differentially expressed in A549 cell models compared with those in controls (Fig. 2D and E). To further validate this result, we tested these ncRNAs on MRC-5 cells derived from human embryonic lung fibroblast cells (Fig. 2F and G). All results are in agreement with those in A549 cell models (12). 
A

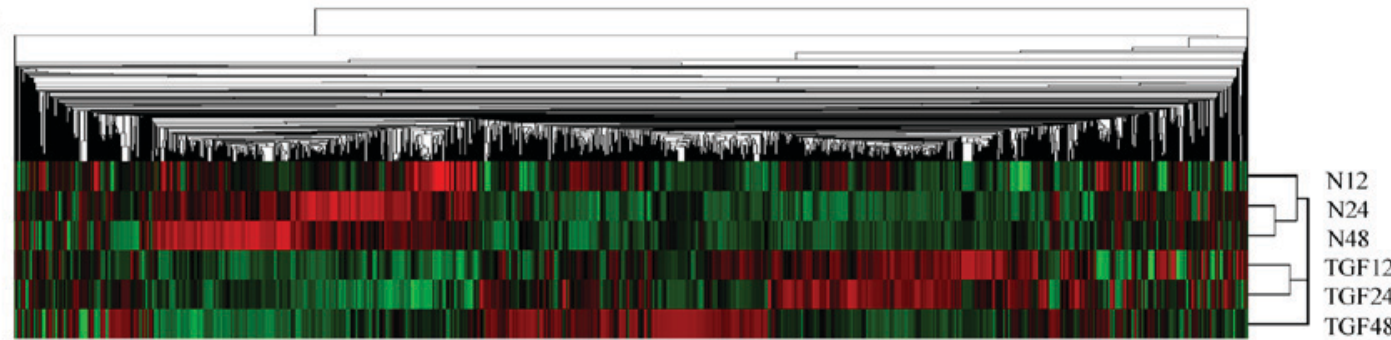

B

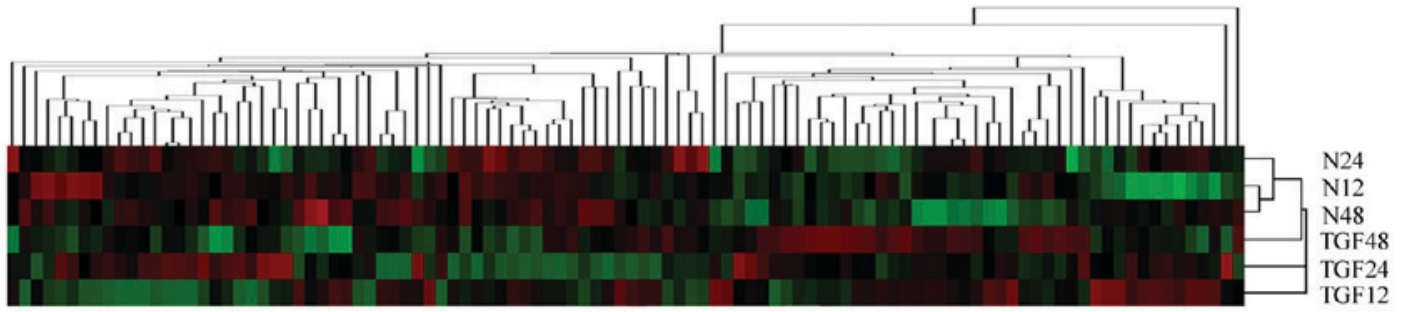

$\mathrm{C}$

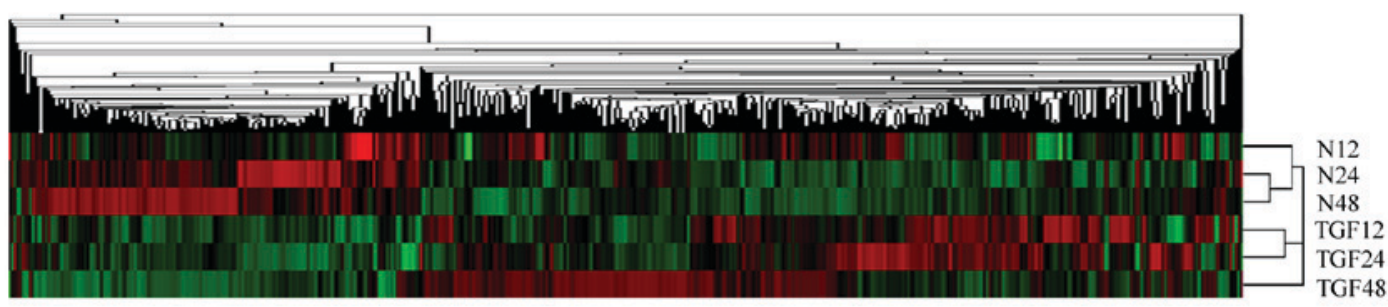

D

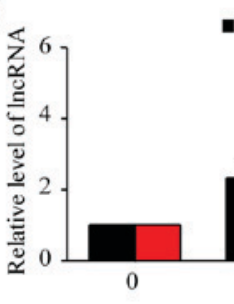

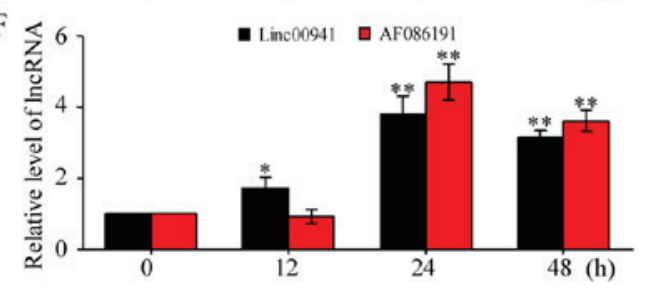

- Linc00941 a AF086191

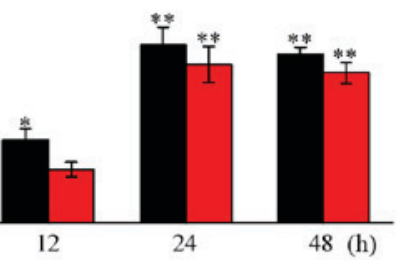

G

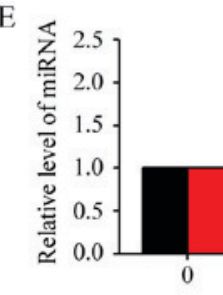

- miR500a-5p amiR628-3p

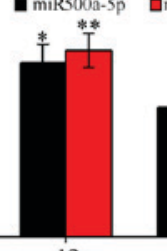

12

- miR500a-5p amiR628-3p

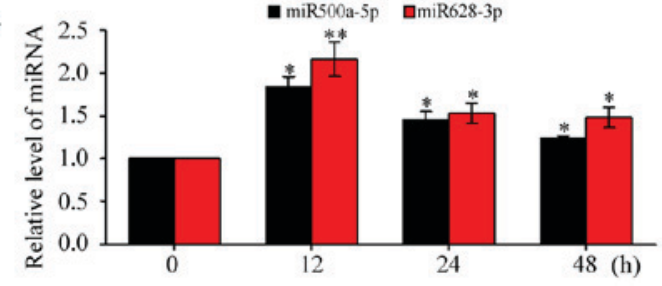

Figure 2. Hierarchical clustering and validation of microarray data. (A-C) Hierarchical clustering of mRNAs (A), miRNAs (B), and lncRNAs (C) in TGF- $\beta 1$-activated pulmonary epithelial cells and normal controls at 12,24 , and $48 \mathrm{~h}$. TGF12-48: TGF- $\beta 1$-activated 12,24 , and $48 \mathrm{~h}$ A549 cell samples. N12-48: Normal A549 cell controls. The red and green shades indicate the expression above and below the relative expression, respectively, across all samples. (D-G) Validation of microarray data by qRT-PCR analysis. Two lncRNAs (D and F) and two miRNAs (E and G) were validated in A549 and MRC-5 cell, respectively. U6 served as an internal control for miRNA, and glyceraldehyde 3-phosphate dehydrogenase for lncRNA. The relative expression level of each miRNA or lncRNA was a normalized one, and data displayed in histograms are expressed as means $\pm \mathrm{SD},{ }^{*} \mathrm{P}<0.05$, ${ }^{* *} \mathrm{P}<0.01 \mathrm{vs}$. control. miRNAs, microRNAs; lncRNAs, long noncoding RNAs; TGF, tumor growth factor.

As expected, we also identified previously discovered fibrotic miR-30a $(28,29)$, thus validating our microarray data.

Analysis of coexpressed mRNAs. The functional classification of 242 coexpressed mRNAs in TGF- $\beta 1$-activated 24 and $48 \mathrm{~h}$ cell samples was analyzed using GO analysis. According to the GO annotation tool, differentially upregulated coexpressed mRNAs were principally enriched for GO terms related to collagen catabolism, cell-matrix adhesion, protein amino acid dephosphorylation, cytoskeleton organization and biogenesis, cell-substrate junction assembly, cell differentiation, response to hypoxia, DNA replication, substrate-bound cell migration, and protein amino acid O-linked glycosylation (Fig. 3A). At the same time, we compared the downregulated coexpression mRNAs in TGF- $\beta 1$-activated 24 and $48 \mathrm{~h}$ cell samples with that in the normal control group, which were principally enriched for GO terms related to ion transport, signal transduction, electron transport chain, proteolysis, oxidation reduction, induction of apoptosis by extracellular signals, inflammatory response, Jun-N-terminal kinase cascade, protein kinase cascade, and apoptosis (Fig. 3B). The TGF- $\beta 1$ induction in EMT-related genes in pulmonary epithelial cells contributes to the upregulation or downregulation of the coexpression of these genes by endowing cells with a specific adhesive, motile, and invasive potential.

Biological pathways associated with coexpressed mRNAs in TGF- $\beta 1$-activated 24 and $48 \mathrm{~h}$ cell samples were analyzed 
A

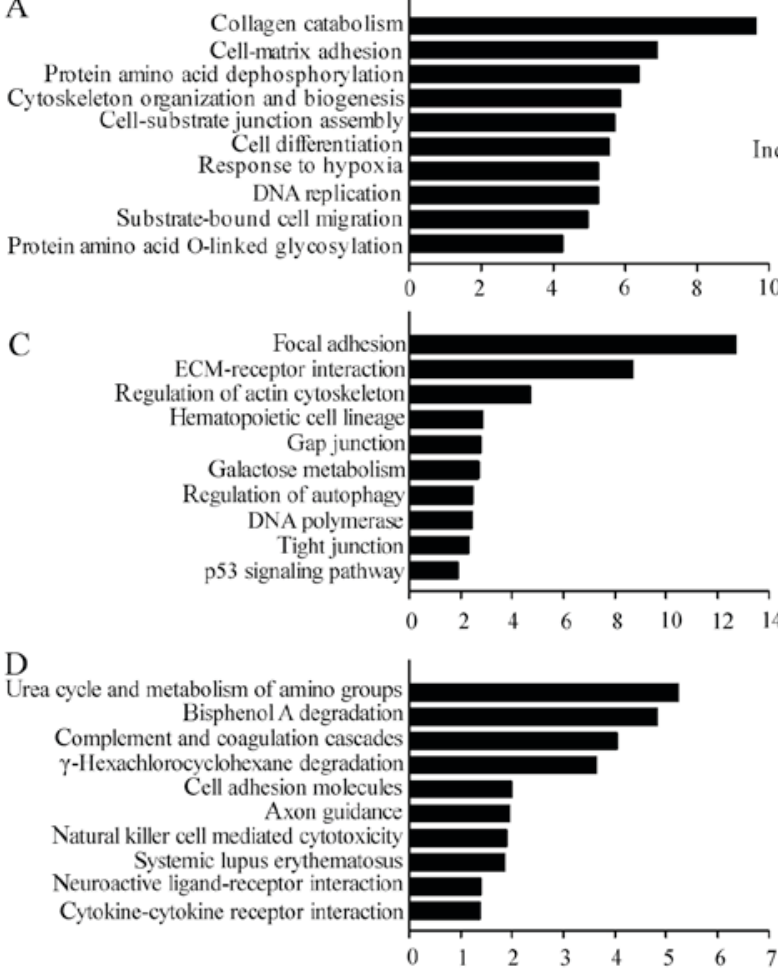

B

nduction of apoptosis by extracellular signals

Inflammatory response

JNK cascad inase cascad Apoptosis

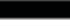

E

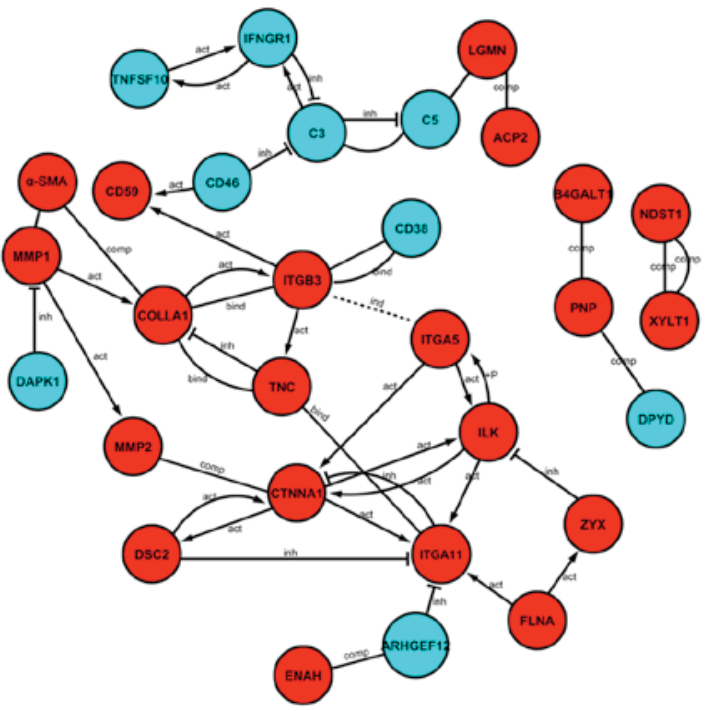

Figure 3. Analysis of coexpressed mRNAs in TGF- $\beta 1$-activated A549 cell samples at 24 and 48 h. Functional classification of the top 10 upregulated (A) and downregulated (B) coexpressed mRNAs using GO analysis. Analysis of the biological upregulated (C) and downregulated (D) pathways associated with coexpressed mRNAs by using the KEGG database. (E) mRNA-mRNA interaction network of coexpressed mRNAs in TGF- $\beta 1$-activated 24 and 48 h cell samples. Upregulated mRNAs are marked red, and downregulated mRNAs are marked blue. Act stands for activated, inh for inhibited; comp for complementary, and bind for binding. TGF- $\beta 1$, tumor growth factor- $\beta 1$.

using the KEGG database (Fig. 3C and D). The 10 upregulated and 10 downregulated pathways listed were identified and associated with focal adhesion, extracellular cell matrix (ECM)-receptor nitration, regulation of actin cytoskeleton, hematopoietic cell lineage, gap junction, galactose metabolism, autophagy regulation, p53 signaling pathway, DNA polymerase, tight junction, and cell adhesion molecules.

Furthermore, we further analyzed the interaction network of the 242 coexpressed mRNAs on the basis of protein-protein interaction. In total, 21 upregulated mRNAs and nine downregulated mRNAs were included in the interaction network (Fig. 3E), which showed four types of interaction, namely, activated, inhibited, complementary, and binding interaction, among the coexpressed 30 mRNAs. CTNNA, ITGA11, and ITGB3 are the network nodes or connections in the interaction network. In addition, MMP1, MMP2, and COLLA1, which are the physiological indicators of fibrogenesis during EMT progression, were also highly and positively expressed.

Coexpressed mRNA-miRNA-lncRNA interaction network. The 30 coexpressed mRNAs were further analyzed. Among these mRNAs, 24 mRNAs, which can coexpress and interact with 11 miRNAs and 33 lncRNAs, exist. The coexpressed interaction network indicated that one mRNA might interact with several miRNAs or lncRNAs. Similarly one miRNA or lncRNA can interact with several mRNAs or lncRNAs (Fig. 4). The degree of mRNA-miRNA-lncRNA coexpression were analyzed. An increased degree indicated an increased coexpression in the mRNA-miRNA-lncRNA interaction network. The coexpressed degree of the mRNAs, miRNAs, and lncRNAs in the network nodes and connections were higher than that of the others (Fig. 5A-C). We further analyzed the lncRNA category. The data showed that most of differentially coexpressed lncRNAs are transcribed from the intergenic gene. Some are from sense-overlapping gene. Meanwhile, a few other unknown sources of lncRNAs exist (Fig. 5D).

In the interaction network, miR-223-3p displays the highest coexpression degree compared with other miRNAs. miR-30a has relatively high coexpression degree, whereas miR-128-3p has relatively low coexpression degree (Fig. 5A). Thus, we selected the three miRNAs and determined their expression levels by using qRT-PCR to illuminate the interaction network. As shown in Fig. 5E, the three miRNAs were differentially decreased in the A549 cell models compared with those in controls. To further validate the data, we tested these miRNA expression levels on MRC-5 cells. The expression trend of these miRNAs is similar pattern to those of miRNAs in A549 cell models (Fig. 5F). Then, analysis of the Pearson's correlation coefficient demonstrated that miR-30a is inversely correlated with collagen I, and miR-128-3p and miR-223-3p are inversely correlated with $\alpha$-SMA (Fig. 5G-I).

Some researchers named the interaction among mRNA, miRNA, and IncRNA functions ceRNA mechanism. A detailed study should be carried out for determining whether the ceRNA mechanism is involved in the interactions among coexpressed mRNAs, miRNAs, and lncRNAs. 


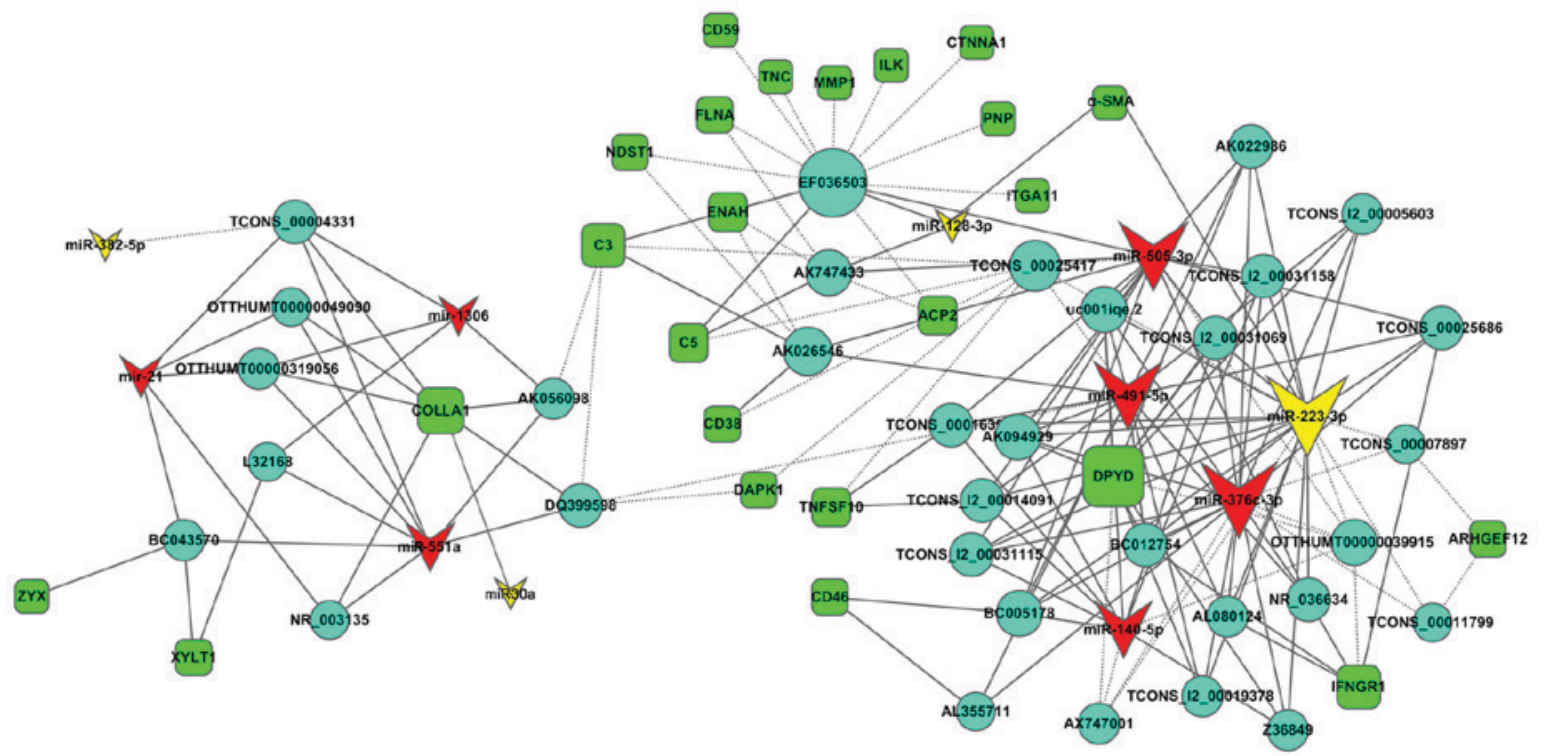

Figure 4. Coexpression of the mRNA-miRNA-lncRNA interaction network. (A) Coexpression of the mRNA-miRNA-lncRNA interaction network in TGF- $\beta 1$-activated A549 cell samples at 24 and 48 h. mRNAs are marked green. Upregulated miRNAs are marked red. Downregulated miRNAs are marked yellow, and lncRNAs are marked blue. miRNA, microRNA; lncRNA, long noncoding RNA.
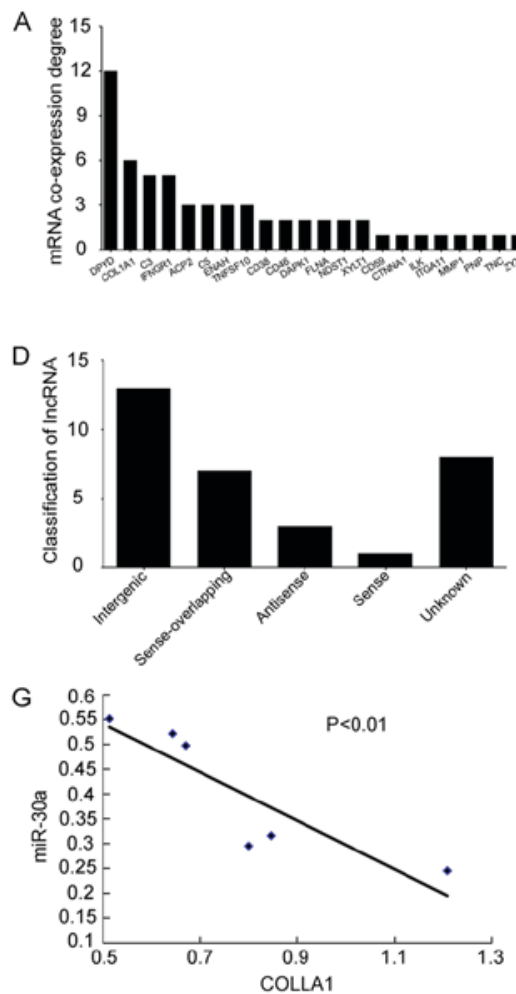
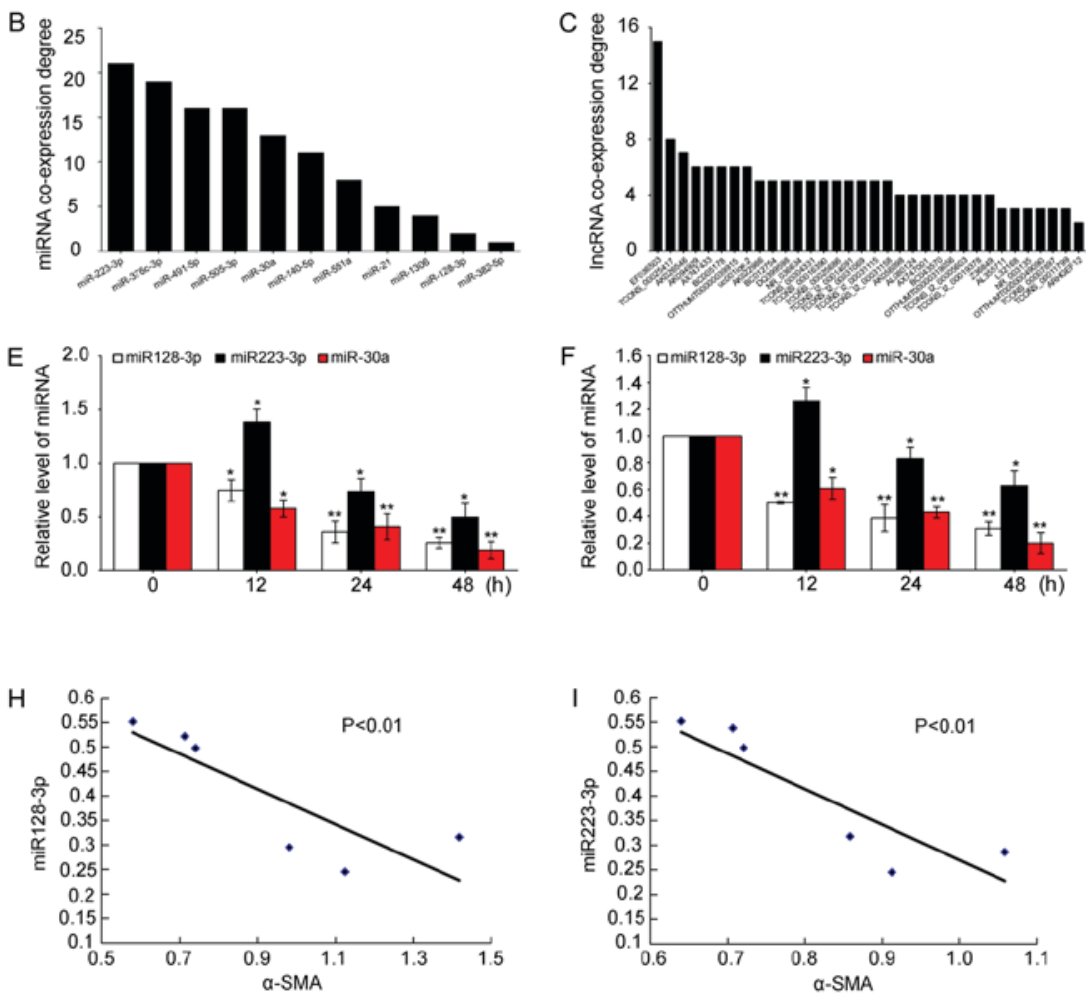

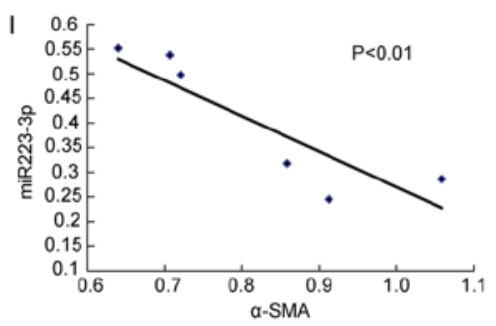

Figure 5. Analysis of the mRNA-miRNA-lncRNA interaction network. (A-C) Coexpression degree of mRNA, miRNA, and lncRNA in TGF- $\beta 1$-activated A549 cell samples at 24 and $48 \mathrm{~h}$. (D) Bar diagram shows the 33 lncRNA category. Most differentially expressed lncRNAs originated from intergenic genes. Some of the lncRNAs were derived from sense overlapping and few from other unknown sources. (E) Expression of miR-30a, miR-128-3p, and miR-223-3p decreased in TGF- $\beta 1$-activated A549 cell samples at 12, 24, and 48 h, respectively. U6 served as an internal control for miRNA. (F) Expression of miR-30a, miR-128-3p, and miR-223-3p decreased in TGF- $\beta 1$-activated MRC-5 cell samples at 12, 24, and $48 \mathrm{~h}$, respectively. U6 served as an internal control for miRNA. (G-I) Pearson correlation coefficient showed that miR-30a, miR-128-3p and miR-223-3p are inversely correlated with collagen I and $\alpha$-SMA, respectively. Data are expressed as mean $\pm \mathrm{SD},{ }^{*} \mathrm{P}<0.05,{ }^{* *} \mathrm{P}<0.01$ vs. control. miRNA, microRNA; lncRNA, long noncoding RNA; TGF- $\beta 1$, tumor growth factor- $\beta 1$.

Interaction network of coexpressed miRNA and targeted genes. The ceRNA mechanism proposed that all types of RNA transcripts communicate through a new 'language' mediated by miRNA-binding sites. The miRNAs bind to sequences with partial complementarity on target RNA transcripts resulting in the repression of target gene expression (17). Thus, we further analyzed the miRNAs and their targeted genes in the interaction network of mRNAs, miRNAs, and lncRNAs. First, the 
A

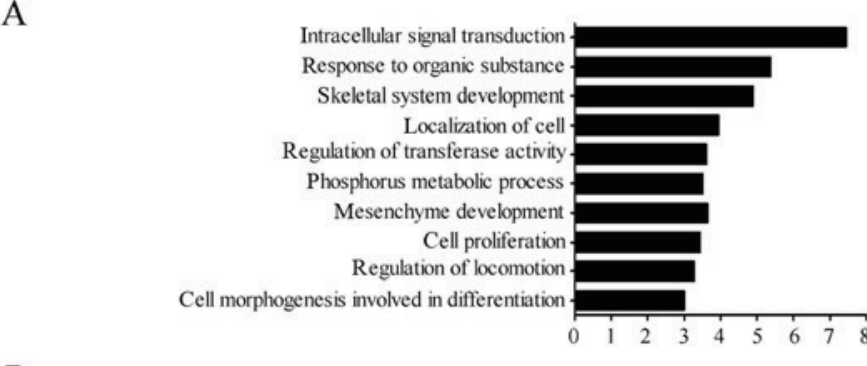

$\mathrm{B}$ Positive regulation of nitrogen compound metabolic process Positive regulation of developmental process Circulatory system development Negative regulation of cell differentiation Response to organic substance Cell projection organization Response to endogenous stimulus Positive regulation of molecular function Cell surface receptor signaling pathway Cell fate commitment

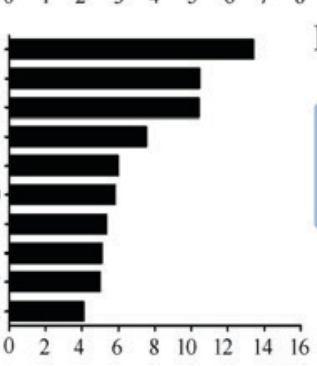

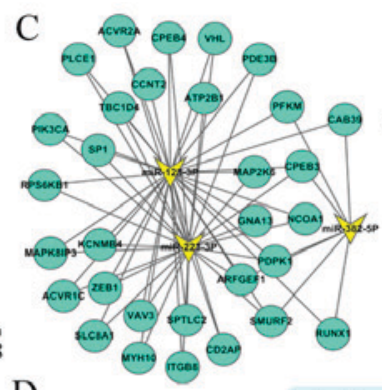
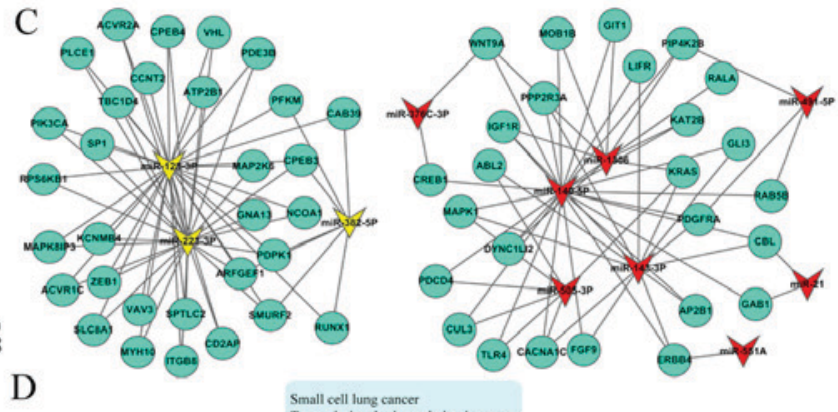

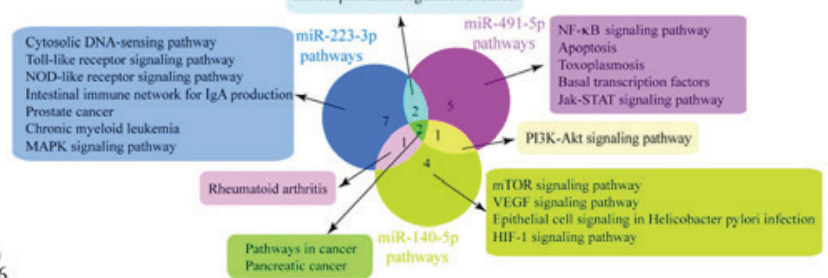

Figure 6. Illustration of miRNAs and their targeted genes. Analysis of the functional classifications of coexpressed miRNA target genes. (A) Upregulated and (B) downregulated target genes. (C) Ten miRNAs and their targeted genes were predicted based on TargetScan, miRanda data, and miRbase. mRNAs are marked blue; upregulated miRNAs are marked red; and downregulated miRNAs are marked yellow. (D) Venn diagram indicates the relevant disease pathways of miR-223-3p mediated, miR-491-5p mediated, and miR-140-5p mediated. Pathways in cancer and pancreatic cancer signaling pathway are intersected. miRNAs, microRNAs

target genes of coexpressed miRNA were predicted based on TargetScan, miRanda data, and miRbase. Subsequently, we analyzed the functional classification of these target genes by GO analysis. The results showed that the upregulated target genes were principally enriched for GO terms related to organic substance response, skeletal system development, transferase activity regulation, mesenchyme development, cell proliferation, and cell morphogenesis (Fig. 6A). The downregulated coexpressed miRNA target genes were principally enriched for GO terms related to the positive regulation of development, negative regulation of cell differentiation, positive regulation of molecular function, and cell fate commitment (Fig. 6B). Most of these upregulated or downregulated miRNA target genes are associated with EMT.

In the interaction network of coexpressed miRNAs and their targeted genes, miR-223-3p, miR-376C-3p, miR-491-5p, miR-505-3p, miR-140-5p, miR-551A, miR-21, miR-1306, miR-128-3p, and miR-382-5p from the mRNA-miRNA-lncRNA interaction network were considered as examples (Fig. 6C). One miRNA had many target genes, whereas different miRNAs can have the same target gene. One gene also can have different binding sites with miRNAs. In total, $57 \mathrm{mRNAs}$ might be the 10 miRNAs' targeted genes. However, among these targeted genes, not a single one is the same as the mRNA in mRNA-miRNA-lncRNA interaction network. This finding means that the ceRNA mechanism does not exist in the above coexpressed mRNA-miRNA-IncRNA interaction network. Analysis with DIANA-miRPath determined 10 candidate miRNAs, which were involved in possible disease-related pathways ( $\mathrm{P}$-value cutoff at 0.05). miR-223-3p, miR-491-5p, and miR-140-5p were significantly implicated in disease-related pathways and were found to be associated in 12,10, and eight pathways, respectively. The Venn diagram revealed the intersection (Fig. 6D). Thus, cancer and pancreatic cancer pathways are crossing pathways related to $\mathrm{miR}-223-3 \mathrm{p}$, miR-491-5p, and miR-140-5p.
In addition, our previous study demonstrated that miR-30a may function as a novel therapeutic target for lung fibrosis by blocking mitochondrial fission dependent on dynamin-related protein1 (Drp-1). miR-30a exerts its regulatory function through regulating Drp-1 promoter hydroxymethylation by directly targeting TET1. To date, our further detailed study has showed no existence of ceRNA mechanism in miR-30a regulatory mode $(28,29)$.

\section{Discussion}

A recent study found that pulmonary fibrosis exhibited several cancer-like pathogenic features. Thus, pulmonary fibrosis is considered to be a neoproliferative disorder of the lung (31). Similar to cancer cells, myofibroblasts show epigenetic and genetic abnormalities, as well as functional features, such as uncontrolled proliferation, resistance to apoptosis, and high migration rates (32). Recently, noncoding RNAs, such as miRNA and lncRNA, have been found to be involved in the development of diseases, especially tumor and pulmonary fibrosis (33-36). However, the mechanism underlying the coexpression and interaction among mRNA, miRNA, and lncRNA in disease regulation requires elucidation. In the present study, we applied microarray and experimental data to examine mRNA, miRNA, and lncRNA coexpression profiles in human alveolar epithelial cells and interpret their interactions. The results indicated that the abnormally expressed mRNA, miRNA, and lncRNA function in the development of pulmonary tumor or fibrosis.

In our previous studies on the EMT process, $10 \mathrm{ng} / \mathrm{ml}$ of TGF- $\beta 1$-activated 24 and $48 \mathrm{~h}$ cell samples were selected $(37,38)$. The coexpression profiles of mRNA, miRNA, and lncRNA in TGF- $\beta 1$-activated 24 and $48 \mathrm{~h}$ cell samples were first investigated in this study. Some studies already reported coexpressed mRNAs or miRNAs, such as COL1 (39), C3 (40), MMP1 (41), miR-30a (42), and miR-21 (43), that can regulate EMT induced by TGF- $\beta$. However, these studies 
usually only focus on targeting a single gene or protein in a single molecular pathway rather than on systematic or coordinated expression of other genes during disease development. Our data showed that the future challenge for an effective pathway-based therapy is how to disrupt numerous altered disease pathways. One specific mRNA, miRNA, or IncRNA may target multiple genes potentially implicated in diseases.

Detailed information on the biological functions and potential mechanisms of 242 mRNAs in EMT based on the results of GO and KEGG pathways were obtained. Then, 29 mRNAs, which were coexpressed and interacted with one another, were ranked. Many of these genes are well known because they often occur in the majority of fibrotic diseases and in various tumors $(44,45)$. These genes can be categorized according to their functions recorded in the KEGG database during EMT. $\mathrm{MMP}_{1}, \mathrm{MMP}_{2}$, and $\mathrm{COL}_{1} \mathrm{~A}_{1}$ were associated with ECM-receptor interaction pathway; ITGA ${ }_{5}, \mathrm{ITGA}_{11}, \mathrm{ITGB}_{3}$, and $\mathrm{CTNNA}_{1}$, with focal adhesion pathway; and $\mathrm{CD}_{38}, \mathrm{CD}_{46}, \mathrm{CD}_{59}, \mathrm{C}_{3}$, and $\mathrm{C}_{5}$, with hematopoietic cell lineage pathway. Thus, the hematopoietic cell plays an important role in human activities, and drawing blood becomes simpler and easier than obtaining tissue samples. As a result, the biomarkers from peripheral blood have attracted considerable attention from researchers investigating diseases, such as tumor and fibrosis (46-48). Our laboratory group reported that the apoptosis of alveolar epithelial cells is suppressed by high miR-30a expression, which can be detected in the peripheral blood of patients with idiopathic pulmonary fibrosis $(28,29)$. Biomarkers or key genes from peripheral blood ideally providing predictive information for the diagnosis, progression, treatment responses, and disease prognosis are thus prospects of future disease research. The high-throughput analysis of large-scale gene data, such as those presented in this study can facilitate the identification of maximally upregulated and downregulated genes, which are possible future targets (49).

Basing on the 29 coexpression mRNAs, we analyzed which coexpression miRNAs and lncRNAs can interact. The results showed that 24 mRNAs, 11 miRNAs, and 33 lncRNAs, can coexpress and interact with one another. Experimental evidence supports that lncRNAs act as ceRNAs for miRNAs and play roles in physiological and pathological processes. Kumar (50) reported that HMGA2 can operate as a ceRNA for the let-7 family to promote lung cancer progression. Karreth (51) also reported that the BRAF pseudogene functions as a ceRNA and induces lymphoma in vivo. Therefore, we further analyzed whether ceRNA is present in the mRNA-miRNA-lncRNA interaction network. Data showed that 57 mRNAs might be the targeted genes of 10 miRNAs. However, no mRNA comes from the mRNA-miRNA-lncRNA interaction network. This finding suggests that no ceRNA exists in the mRNA-miRNA-lncRNA interaction network. Living beings have evolved complex mechanisms for efficient functioning. ceRNA theories can only illustrate the possibility of regulation in living beings. Moreover, some other functional modes, such as histone modification and transcription factor recruitment in the interaction among mRNA, miRNA, and lncRNA, occur. Wang (52) reported that lncRNA-DC promotes STAT3 signaling by interacting with the $\mathrm{C}$ terminus of STAT3 to control human dendritic cell differentiation. However, the possibility of ceRNA existing in pulmonary tumor and fibrosis cannot be ruled out because of the generally low abundance of IncRNAs, and the frequent promiscuity of protein-RNA interactions. Of course, this research is still in the exploratory stage, and conclusions were obtained only through the changes in their levels and bioinformatics analysis, needing further experimental identification and validation.

The mRNA-miRNA-IncRNA interaction network showed that during the occurrence and development of diseases, many mRNA, miRNA, or lncRNA molecules are involved. The term interaction means different things. Proteins encoded by the mRNAs may interact with each other, with miRNAs, or with lncRNAs. miRNAs may interact and regulate the stability or expression of mRNAs or lncRNAs. lncRNAs may interact with miRNAs or mRNAs. Each gene regulates a broad spectrum of target genes and affects many signaling pathways. Therefore, disruption of a single gene expression may only limit an inhibitory effect, whereas joint interference of multiple genes may be effective. Thus, determining how to silence multiple abnormally expressed genes simultaneously in order to enhance the efficacy of disease treatments is important. Li (53) reported an artificially designed interfering lncRNA, which simultaneously contains sequences that can complementarily bind to multiple miRNAs.

The genes enlisted in the current study represent only a small proportion of genes published on pulmonary tumor or fibrosis. This condition may be correlated with the time point of analysis or the specific condition of in vitro culture of the cells. In conclusion, the present study provided new information regarding the potential role of mRNAs, miRNAs, and lncRNAs in EMT. These findings may provide basic mechanistic information, possible biomarkers, and novel treatment of EMT-targeted strategies for diseases, especially for pulmonary tumor and fibrosis.

\section{Acknowledgements}

This study was supported by National Natural Science Foundation of China (31670365, 31470415, 81670064), Important Project of Science and Technology of Shandong Province (2014GSF119014), Project of Science and Technology of Yantai (2016ZH082). International Cooperation Program for Key Professors by Shandong Provincial Education Deparment.

\section{References}

1. Fernandez IE and Eickelberg O: New cellular and molecular mechanisms of lung injury and fibrosis in idiopathic pulmonary fibrosis. Lancet 380: 680-688, 2012.

2. Alonso SR, Tracey L, Ortiz P, Pérez-Gómez B, Palacios J, Pollán M, Linares J, Serrano S, Sáez-Castillo AI, Sánchez L, et al: A high-throughput study in melanoma identifies epithelial-mesenchymal transition as a major determinant of metastasis. Cancer Res 67: 3450-3460, 2007.

3. Oruqaj G, Karnati S, Vijayan V, Kotarkonda LK, Boateng E, Zhang W, Ruppert C, Günther A, Shi W and Baumgart-Vogt E: Compromised peroxisomes in idiopathic pulmonary fibrosis, a vicious cycle inducing a higher fibrotic response via TGF- $\beta$ signaling. Proc Natl Acad Sci USA 112: 2048-2057, 2015.

4. Akhurst RJ and Hata A: Targeting the TGF $\beta$ signalling pathway in disease. Nat Rev Drug Discov 11: 790-811, 2012.

5. Blackwell TS, Tager AM, Borok Z, Moore BB, Schwartz DZ, Anstom KJ, Bar-Joseph Z, Bitterman P, Blackburn MR, Bradford W, et al: Future directions in idiopathic pulmonary fibrosis research. An NHLBI workshop report. Am J Respir Crit Care Med 189: 214-222, 2014. 
6. Iser IC, Pereira MB, Lenz G and Wink MR: The epithelial-to-mesenchymal transition-like process in glioblastoma: An updated systematic review and in silico investigation. Med Res Rev 37: 271-313, 2017

7. Shenoy A and Blelloch RH: Regulation of microRNA function in somatic stem cell proliferation and differentiation. Nat Rev Mol Cell Biol 15: 565-576, 2014.

8. Holdt LM, Hoffmann S, Sass K, Langenberger D, Scholz M, Krohn K, Finstermeier K, Stahringer A, Wilfert W, Beutner F, et al: Alu elements in ANRIL non-coding RNA at chromosome 9p21 modulate atherogenic cell functions through trans-regulation of gene networks. PLoS Genet 9: e1003588, 2013.

9. Payer B, Rosenberg M, Yamaji M, Yabuta Y, Koyanagi-Aoi M, Hayashi K, Yamanaka S, Saitou M and Lee JT: Tsix RNA and the germline factor, PRDM14, link X reactivation and stem cell reprogramming. Mol Cell 52: 805-818, 2013.

10. Song R, Walentek P, Sponer N, Klimke A, Lee JS, Dixon G, Harland R, Wan Y, Lishko P, Lize M, et al: miR-34/449 miRNAs are required for motile ciliogenesis by repressing cp110. Nature 510: 115-120, 2014.

11. Nicodemus-Johnson J, Laxman B, Stern RK, Sudi J, Tierney CN, Norwick L, Hogarth DK, McConville JF, Naureckas ET, Sperling AI, et al: Maternal asthma and microRNA regulation of soluble HLA-G in the airway. J Allergy Clin Immunol 131: 1496-1503, 2013

12. Caruso P, Dempsie Y, Stevens HC, McDonald RA, Long L, Lu R, White K, Mair KM, McClure JD, Southwood M, et al: A role for miR-145 in pulmonary arterial hypertension: Evidence from mouse models and patient samples. Circ Res 111: 290-300, 2012.

13. Sayed D and Abdellatif M: MicroRNAs in development and disease. Physiol Rev 91: 827-887, 2011.

14. Sun Q, Csorba T, Skourti-Stathaki K, Proudfoot NJ and Dean C: R-loop stabilization represses antisense transcription at the Arabidopsis FLC locus. Science 340: 619-621, 2013

15. Tollervey JR, Curk T, Rogelj B, Briese M, Cereda M, Kayikci M, König J, Hortobágyi T, Nishimura AL, Zupunski V, et al: Characterizing the RNA targets and position-dependent splicing regulation by TDP-43. Nat Neurosci 14: 452-458, 2011.

16. Gontan C, Achame EM, Demmers J, Barakat TS, Rentmeester E, van IJcken W, Grootegoed JA and Gribnau J: RNF12 initiates $\mathrm{X}$-chromosome inactivation by targeting REX1 for degradation. Nature 485: 386-390, 2012

17. Salmena L, Poliseno L, Tay Y, Kats L and Pandolfi PP: A ceRNA hypothesis: The Rosetta Stone of a hidden RNA language? Cell 146: 353-358, 2011.

18. Kino T, Hurt DE, Ichijo T, Nader N and Chrousos GP: Noncoding RNA gas5 is a growth arrest- and starvation-associated repressor of the glucocorticoid receptor. Sci Signal 3: ra8, 2010.

19. Liu X, Li D, Zhang W, Guo M and Zhan Q: Long non-coding RNA gadd7 interacts with TDP-43 and regulates Cdk6 mRNA decay. EMBO J 31: 4415-4427, 2012.

20. Hung T, Wang Y, Lin MF, Koegel AK, Kotake Y, Grant GD, Horlings HM, Shah N, Umbricht C, Wang P, et al: Extensive and coordinated transcription of noncoding RNAs within cell-cycle promoters. Nat Genet 43: 621-629, 2011.

21. Lee S, Kopp F, Chang TC, Sataluri A, Chen B, Sivakumar S, Yu H, Xie Y and Mendell JT: Noncoding RNA NORAD regulates genomic stability by sequestering PUMILIO proteins. Cell 164: 69-80, 2016

22. Cao G, Zhang J, Wang M, Song X, Liu W, Mao C and Lv C: Differential expression of long non-coding RNAs in bleomycin-induced lung fibrosis. Int J Mol Med 32: 355-364, 2013.

23. Song X, Cao G, Jing L, Lin S, Wang X, Zhang J, Wang M, Liu W and Lv C: Analysing the relationship between lncRNA and protein-coding gene and the role of IncRNA as ceRNA in pulmonary fibrosis. J Cell Mol Med 18: 991-1003, 2014.

24. Foster KA, Oster CG, Mayer MM, Avery ML and Audus KL: Characterization of the A549 cell line as a type II pulmonary epithelial cell model for drug metabolism. Exp Cell Res 243: 359-366, 1998

25. Vockley CM, D'Ippolito AM, McDowell IC, Majoros WH, Safi A, Song L, Crawford GE and Reddy TE: Direct GR binding sites potentiate clusters of TF binding across the human genome. Cell 166: 1269-1281, 2016.

26. Pardo A, Gibson K, Cisneros J, Richards TJ, Yang Y, Becerril C, Yousem S, Herrera I, Ruiz V, Selman M and Kaminski N: Up-regulation and profibrotic role of osteopontin in human idiopathic pulmonary fibrosis. PLoS Med 2: e251, 2005.
27. Roberts JR, Perkins GD, Fujisawa T, Pettigrew KA, Gao F, Ahmed A and Thickett DR: Vascular endothelial growth factor promotes physical wound repair and is anti-apoptotic in primary distal lung epithelial and A549 cells. Crit Care Med 35: 2164-2170, 2007.

28. Mao C, Zhang J, Lin S, Jing L, Xiang J, Wang M, Wang B, Xu P, Liu W, Song X and Lv C: miR-30a inhibits AECs-II apoptosis by blocking mitochondrial fission dependent on Drp-1. J Cell Mol Med 18: 2404-2416, 2014.

29. Zhang S, Liu H, Liu Y, Zhang J, Li H, Liu W, Cao G, Xv P, Zhang J, Lv C and Song X: miR-30a as potential therapeutics by targeting TET1 through regulation of Drp-1 promoter hydroxymethylation in idiopathic pulmonary fibrosis. Int J Mol Sci 18: pii: E633, 2017.

30. Zhang YL: The value of disease. Guangxi Normal University Press, Guilin, 2014

31. Vancheri C, Failla M, Crimi N and Raghu G: Idiopathic pulmonary fibrosis: A disease with similarities and links to cancer biology. Eur Respir J 35: 496-504, 2010.

32. Vancheri C: Idiopathic pulmonary fibrosis: An altered fibroblast proliferation linked to cancer biology. Proc Am Thorac Soc 9: $153-157,2012$.

33. Huang C, Yang Y and Liu L: Interaction of long noncoding RNAs and microRNAs in the pathogenesis of idiopathic pulmonary fibrosis. Physiol Genomics 47: 463-469, 2015.

34. Sun H, Chen J, Qian W, Kang J, Wang J, Jiang L, Qiao L, Chen W and Zhang J: Integrated long non-coding RNA analyses identify novel regulators of epithelial-mesenchymal transition in the mouse model of pulmonary fibrosis. J Cell Mol Med 20: $1234-1246,2016$

35. Yue B, Cai D, Liu C, Fang C and Yan D: Linc00152 functions as a competing endogenous RNA to confer oxaliplatin resistance and holds prognostic values in colon cancer. Mol Ther 24: 2064-2077, 2016

36. Xing Z, Lin A, Li C, Liang K, Wang S, Liu Y, Park P, Qin L, Wei Y, Hawke D, et al: lncRNA directs cooperative epigenetic regulation downstream of chemokine signals. Cell 159: $1110-1125,2014$

37. Zhang J, Xv P, Wang Y, Wang M, Li H, Lin S, Mao C, Wang B, Song $\mathrm{X}$ and Lv C: Astaxanthin prevents pulmonary fibrosis by promoting myofibroblast apoptosis dependent on Drp1-mediated mitochondrial fission. J Cell Mol Med 19: 2215-2231, 2015.

38. Wang M, Zhang J, Song X, Liu W, Zhang L, Wang X and Lv C: Astaxanthin ameliorates lung fibrosis both in vivo and in vitro through preventing transdifferentiation, inhibiting proliferation, and promoting apoptosis of activated cells. Food Chem Toxicol 56: 450-458, 2013.

39. Alipio ZA, Jones N, Liao W, Yang J, Kulkarni S, Sree Kumar K, Hauer-Jensen M, Ward DC, Ma Y and Fink LM: Epithelial to mesenchymal transition (EMT) induced by bleomycin or TFG(b1)/EGF in murine induced pluripotent stem cell-derived alveolar Type II-like cells. Differentiation 82: 89-98. 2011

40. Cho MS, Rupaimoole R, Choi HJ, Noh K, Chen J, Hu Q, Sood AK and Afshar-Kharghan V: Complement component 3 is regulated by TWIST1 and mediates epithelial-mesenchymal transition. J Immunol 196: 1412-1418, 2016.

41. Uttamsingh S, Bao X, Nguyen KT, Bhanot M, Gong J, Chan JL, Liu F, Chu TT and Wang LH: Synergistic effect between EGF and TGF-beta1 in inducing oncogenic properties of intestinal epithelial cells. Oncogene 27: 2626-2634, 2008.

42. Kao CJ, Martiniez A, Shi XB, Yang J, Evans CP, Dobi A, deVere White RW and Kung HJ: miR-30 as a tumor suppressor connects EGF/Src signal to ERG and EMT. Oncogene 33: 2495-2503, 2014.

43. Braun J,Hoang-Vu C, Dralle H and Hüttelmaier S: Downregulation of microRNAs directs the EMT and invasive potential of anaplastic thyroid carcinomas. Oncogene 29: 4237-4244, 2010.

44. Selman M, Pardo A, Barrera L, Estrada A, Watson SR, Wilson K, Aziz N, Kaminski N and Zlotnik A: Gene expression profiles distinguish idiopathic pulmonary fibrosis from hypersensitivity pneumonitis. Am J Respir Crit Care Med 173: 188-198, 2006

45. Nakerakanti SS, Kapanadze B, Yamasaki M, Markiewicz M and Trojanowska M: Fli1 and Ets1 have distinct roles in connective tissue growth factor/CCN2 gene regulation and induction of the profibrotic gene program. J Biol Chem 281: 25259-25269, 2006.

46. Noé G, Bellesoeur A, Thomas-Schoemann A, Rangarajan S, Naji F, Puszkiel A, Huillard O, Saidu N, Golmard L, Alexandre $\mathrm{J}$, et al: Clinical and kinomic analysis identifies peripheral blood mononuclear cells as a potential pharmacodynamic biomarker in metastatic renal cell carcinoma patients treated with sunitinib. Oncotarget 7: 67507-67520, 2016. 
47. Fernandez IE, Greiffo FR, Frankenberger M, Bandres J, Heinzelmann K, Neurohr C, Hatz R, Hartl D, Behr J and Eickelberg O: Peripheral blood myeloid-derived suppressor cells reflect disease status in idiopathic pulmonary fibrosis. Eur Respir J 48: 1171-1183, 2016.

48. Roderburg $\mathrm{C}$ and Luedde T: Circulating microRNAs as markers of liver inflammation, fibrosis and cancer. J Hepatol 61: 1434-1437, 2014

49. du Bois RM: Strategies for treating idiopathic pulmonary fibrosis. Nat Rev Drug Discov 9: 129-140, 2010.

50. Kumar MS, Armenteros-Monterroso E, East P, Chakravorty P, Matthews N, Winslow MM and Downward J: HMGA2 functions as a competing endogenous RNA to promote lung cancer progression. Nature 505: 212-217, 2014.
51. Karreth FA, Reschke M, Ruocco A, Ng C, Chapuy B, Léopold V, Sjoberg M, Keane TM, Verma A, Ala U, et al: The BRAF pseudogene functions as a competitive endogenous RNA and induces lymphoma in vivo. Cell 161: 319-332, 2015.

52. Wang P, Xue Y, Han Y, Lin L, Wu C, Xu S, Jiang Z, Xu J, Liu Q and Cao X: The STAT3-binding long noncoding RNA lnc-DC controls human dendritic cell differentiation. Science 344: 310-313, 2014.

53. Li X, Su Y, Sun B, Ji W, Peng Z, Xu Y, Wu M and Su C: An artificially-designed interfering lncRNA expressed by oncolytic adenovirus competitively consumes oncomiRs to exert antitumor efficacy in hepatocellular carcinoma. Mol Cancer Ther 15: 1436-1451, 2016 